\title{
Cerebellum to motor cortex paired associative stimulation induces bidirectional STDP-like plasticity in human motor cortex
}

\author{
Ming-Kuei Lu ${ }^{1,2,3}$, Chon-Haw Tsai ${ }^{2,3}$ and Ulf Ziemann ${ }^{1,4 *}$ \\ ${ }^{1}$ Department of Neurology, Goethe-University, Frankfurt/Main, Germany \\ ${ }^{2}$ Neuroscience Laboratory, Department of Neurology, China Medical University Hospital, Taichung, Taiwan \\ ${ }^{3}$ Institute of Medical Science and School of Medicine, China Medical University, Taichung, Taiwan \\ ${ }^{4}$ Department of Neurology, Hertie-Institute for Clinical Brain Research, University Hospital Tübingen, Tübingen, Germany
}

\section{Edited by:}

Sven Bestmann, University College London, UK

\section{Reviewed by:}

Joseph M. Galea, University College London, UK

Giacomo Koch, Santa Lucia IRCCS, Italy

\section{*Correspondence:}

Ulf Ziemann, Department of

Neurology and Stroke, Hertie

Institute for Clinical Brain Research,

Eberhard-Karls-University Tübingen,

Hoppe-Seyler-Str. 3, D-72076

Tübingen, Germany.

e-mail: ulf.ziemann@med.

uni-tuebingen.de
The cerebellum is crucially important for motor control and adaptation. Recent non-invasive brain stimulation studies have indicated the possibility to alter the excitability of the cerebellum and its projections to the contralateral motor cortex, with behavioral consequences on motor control and adaptation. Here we sought to induce bidirectional spike-timing dependent plasticity (STDP)-like modifications of motor cortex (M1) excitability by application of paired associative stimulation (PAS) in healthy subjects. Conditioning stimulation over the right lateral cerebellum (CB) preceded focal transcranial magnetic stimulation (TMS) of the left $\mathrm{M} 1$ hand area at an interstimulus interval of $2 \mathrm{~ms}$ $\left(\mathrm{CB} \rightarrow \mathrm{M} 1 \mathrm{PAS}_{2 \mathrm{~ms}}\right), 6 \mathrm{~ms}\left(\mathrm{CB} \rightarrow \mathrm{M} 1 \mathrm{PAS}_{6 \mathrm{~ms}}\right)$ or $10 \mathrm{~ms}\left(\mathrm{CB} \rightarrow \mathrm{M} 1 \mathrm{PAS}_{10 \mathrm{~ms}}\right)$ or randomly alternating intervals of 2 and $10 \mathrm{~ms}(\mathrm{CB} \rightarrow \mathrm{M} 1 \mathrm{PAS}$ Control). Effects of PAS on M1 excitability were assessed by the motor-evoked potential (MEP) amplitude, short-interval intracortical inhibition (SICI), intracortical facilitation (ICF) and cerebellar-motor cortex inhibition (CBI) in the first dorsal interosseous muscle of the right hand. $\mathrm{CB} \rightarrow \mathrm{M} 1 \mathrm{PAS}_{2 \mathrm{~ms}}$ resulted in MEP potentiation, $\mathrm{CB} \rightarrow \mathrm{M} 1 \mathrm{PAS}_{6 \mathrm{~ms}}$ and $\mathrm{CB} \rightarrow \mathrm{M} 1 \mathrm{PAS}_{10 \mathrm{~ms}}$ in MEP depression, and $\mathrm{CB} \rightarrow \mathrm{M} 1 \mathrm{PAS}_{\text {Control }}$ in no change. The MEP changes lasted for 30-60 min after PAS. SICl and $\mathrm{CBI}$ decreased non-specifically after all PAS protocols, while ICF remained unaltered. The physiological mechanisms underlying these MEP changes are carefully discussed. Findings support the notion of bidirectional STDP-like plasticity in M1 mediated by associative stimulation of the cerebello-dentato-thalamo-cortical pathway and M1. Future studies may investigate the behavioral significance of this plasticity.

Keywords: cerebello-dentato-thalamo-cortical pathway, spike-timing dependent-like plasticity, cerebellum, motor cortex, transcranial magnetic stimulation, paired associative stimulation, human

\section{INTRODUCTION}

The cerebellum is essentially important for control of posture and movement (Brooks and Thach, 1981) and for specific motor learning processes, in particular motor adaptation (Shmuelof and Krakauer, 2011). The cerebellum has abundant efferent projections to distributed areas of the sensorimotor cortex (Allen and Tsukahara, 1974; Thach, 1987; Middleton and Strick, 1998; Hoover and Strick, 1999) and influences motor behavior through these connections. Excitability of the cerebellum and its cerebellodentato-thalamo-cortical connections can be altered in humans by non-invasive brain stimulation techniques such as transcranial magnetic stimulation (TMS) and transcranial direct current stimulation of the lateral cerebellum (Oliveri et al., 2005; Fierro et al., 2007; Koch et al., 2008; Galea et al., 2009, 2011; Popa et al., 2009). Concomitantly, non-invasive stimulation of the lateral cerebellum can lead to changes in the spatial and timing precision of hand movements (Miall and Christensen, 2004; Del Olmo et al., 2007), adaptive motor learning (Galea et al., 2011; Panouilleres et al., 2012), associative motor learning (Hoffland et al., 2012), and procedural motor learning (Torriero et al., 2004).
Paired associative stimulation (PAS) is a well explored stimulation technique that allows induction of bidirectional spike-timing dependent plasticity (STDP)-like plasticity (for reviews, Ziemann et al., 2008; Müller-Dahlhaus et al., 2010). Depending on the interstimulus interval between an afferent input into the primary motor cortex (M1) and action potential generation in M1 corticospinal neurons by suprathreshold TMS, long-term depression (LTD)-like or long-term potentiation (LTP)-like plasticity of corticospinal neurons occurs, strongly reminiscent of STDP as studied at the level of single cells in brain slices or neuronal cultures (Markram et al., 1997; Bi and Poo, 2001). Bidirectional STDP-like plasticity has so far been demonstrated for repeated pairing of TMS of M1 with afferent inputs into M1 from peripheral nerves (Stefan et al., 2000, 2002; Wolters et al., 2003; Ziemann et al., 2004; Müller et al., 2007) and from the ipsilateral ventral premotor cortex (Buch et al., 2011).

Here we sought to test the possibility to induce STDPlike plasticity along the cerebellar-dentato-thalamo-M1 connection by cerebellum-to-M1 (CB $\rightarrow$ M1) PAS. In doing so, several 
peculiarities of this pathway have to be taken into consideration: TMS of the lateral cerebellum most likely excites Purkinje cells (Ugawa et al., 1995), i.e., the principal inhibitory neurons of the cerebellum. This leads to inhibition of target neurons in the dentate nucleus and, consequently, to disfacilitation of the tonically active bi-synaptic excitatory projection to M1, measurable as "cerebellar-motor inhibition" (CBI) of motor-evoked potentials (MEPs) (Ugawa et al., 1995).

We show that $\mathrm{CB} \rightarrow \mathrm{M} 1$ PAS leads to MEP depression or MEP potentiation, depending on the interstimulus interval between the $\mathrm{CB}$ and M1 stimuli, most likely explained by Hebbian weakening or strengthening, respectively, of the cerebello-dentato-thalamoM1 connection. This bidirectional modification of M1 excitability may prove useful for correcting abnormal M1 excitability caused by cerebellar disease (Groiss and Ugawa, 2012). Also, behavioral effects of $\mathrm{CB} \rightarrow \mathrm{M} 1 \mathrm{PAS}$, in particular on motor performance and various forms of motor learning are possible and warrant further investigation.

\section{METHODS \\ SUBJECTS}

Nineteen healthy subjects participated in this study (mean age \pm SD, $29.8 \pm 6.9$ years; range, $22-42$ years; four female). All subjects were right-handed according to the Edinburgh Handedness Inventory (Oldfield, 1971). All gave their written informed consent prior to the study. The experimental procedures were in accord with the Declaration of Helsinki and approved by the local
Ethics Committee of the medical faculty of the Goethe-University of Frankfurt am Main, Germany.

\section{EXPERIMENTAL DESIGN}

Subjects were seated on a comfortable reclining chair with both arms relaxed. Cortical excitability of the hand representation of the left primary motor cortex $\left(\mathrm{M} 1_{\mathrm{HAND}}\right)$ was tested with singlepulse and paired-pulse TMS in blocks of measurements immediately before $\mathrm{CB} \rightarrow \mathrm{M} 1$ PAS (baseline, B0) and immediately, 30 and 60 min after $\mathrm{CB} \rightarrow \mathrm{M} 1 \mathrm{PAS}$ (P0, P30, and P60, respectively). The target muscle for EMG recordings was the first dorsal interosseus (FDI) of the right hand. The individual resting motor threshold (RMT) and active motor threshold (AMT) were determined over the left $\mathrm{M} 1_{\mathrm{HAND}}$, and AMT was, in addition, determined over the inion prior to baseline. Thresholds were determined to the nearest $1 \%$ of maximum stimulator output (MSO) using the relative frequency method (Rossini et al., 1994; Groppa et al., 2012). RMT was defined as the lowest intensity that was sufficient to elicit MEPs $=50 \mu \mathrm{V}$ in at least 5 of 10 consecutive trials. AMT was tested in the slightly activated FDI $(\sim 10 \%$ of maximum voluntary contraction) and defined as the lowest stimulator intensity to elicit an $\mathrm{MEP}=100 \mu \mathrm{V}$ in the average of five consecutive trials (Ziemann et al., 1996). Left M1 HAND excitability measurements at time points B0, P0, P30, and P60 consisted of MEP amplitude, short-interval intracortical inhibition (SICI), CBI and, in a subset of subjects, intracortical facilitation (ICF) as detailed below (Figure 1). All these measures were obtained in the voluntarily

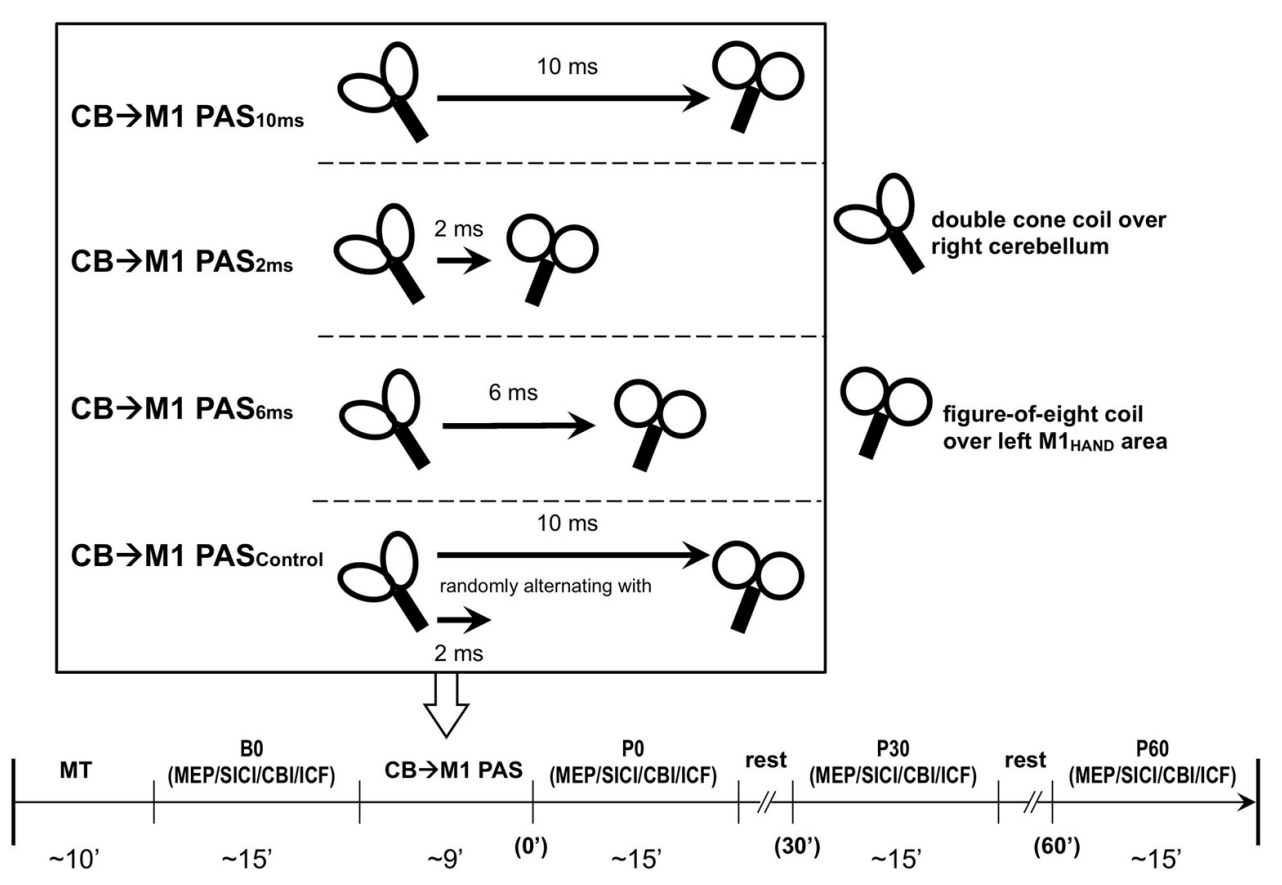

FIGURE 1 | Experimental design and time line of the cerebellum $(\mathrm{CB}) \rightarrow$ motor cortex (M1) paired associative stimulation (PAS) study. Four PAS protocols were applied in healthy subjects: $\mathrm{CB} \rightarrow \mathrm{M} 1 \mathrm{PAS}_{10 \mathrm{~ms}}$ [i.e., interstimulus interval between magnetic pulses to right lateral $\mathrm{CB}$ and hand area of left $\mathrm{M} 1$ (M1 $1_{\text {HAND }}$ ) was $10 \mathrm{~ms}$ ), $\mathrm{CB} \rightarrow \mathrm{M} 1 \mathrm{PAS}_{6 \mathrm{~ms}}$ (interstimulus interval of $6 \mathrm{~ms}$ ), $\mathrm{CB} \rightarrow \mathrm{M} 1 \mathrm{PAS}_{2} \mathrm{~ms}$ (interstimulus interval of $2 \mathrm{~ms}$ ), and $\mathrm{CB} \rightarrow \mathrm{M} 1$
PAS $_{\text {Control }}$ (randomly alternating intervals of 2 and $10 \mathrm{~ms}$ ). The timeline in the lower part of the diagram shows the order of measurements and their approximate durations (in min) before (B0) and after $\mathrm{CB} \rightarrow \mathrm{M} 1 \mathrm{PAS}$ (P0, P30, and P60: immediately and 30 and 60 min post-PAS). Abbreviations: MT, motor threshold; MEP, motor-evoked potential; $\mathrm{SICl}$, short-interval intracortical inhibition; $\mathrm{CBI}$, cerebellar-motor cortex inhibition; ICF, intracortical facilitation. 
relaxed FDI. Maintenance of muscle relaxation was monitored by online audio-visual feedback of high-gain ( $50 \mu \mathrm{V} /$ division) EMG.

\section{MEASUREMENT OF MOTOR-EVOKED POTENTIAL (MEP) AMPLITUDE}

TMS was delivered through a focal figure-of-eight stimulating coil (inner diameter of each wing, $70 \mathrm{~mm}$ ) connected via a BiStim module to two Magstim 200 magnetic stimulators (Magstim Co., Carmarthenshire, Wales, UK) with a monophasic current waveform. The coil was held tangential to the scalp over the presumed hand area of the left M1 with the handle pointing backwards and $\sim 45^{\circ}$ away from the midline, thus inducing a current in M1 from posterior-lateral to anterior-medial which is optimal for activating corticospinal neurons transsynaptically (Di Lazzaro et al., 2008). The optimal coil position ("hot spot"; M1 $1_{\text {HAND }}$ ) was determined as the site where TMS at a slightly suprathreshold intensity produced consistently the largest MEPs in the right FDI. This site was marked on the scalp to ensure a constant placement of the coil throughout the session. At B0, the intensity of TMS was adjusted to produce MEPs of on average $1 \mathrm{mV}$ in peak-to-peak amplitude $\left(\mathrm{MEP}_{1 \mathrm{mV}}\right)$ in the resting FDI. The same intensity was applied throughout all post cerebellum-toM1 PAS ( $\mathrm{CB} \rightarrow \mathrm{M} 1$ PAS) measurements. The MEPs were recorded using $\mathrm{Ag}-\mathrm{AgCl}$ electrodes with the active electrode mounted on the motor point of the FDI and the reference electrode on the proximal phalanx of the index finger. The raw EMG was bandpass filtered $(20 \mathrm{~Hz}-2 \mathrm{kHz}$; Counterpoint Mk2 Electromyograph; Dantec, Skovlunde, Denmark), digitized at a rate of $5 \mathrm{kHz}$ (CED Micro 1401; Cambridge Electronic Design, Cambridge, UK) and stored in a laboratory computer for offline analysis (Spike2 for Windows, Version 3.05, CED). Twenty trials of MEPs were obtained at each time point (B0, P0, P30, and P60). For MEP and all other recordings (SICI, ICF, and $\mathrm{CBI}$ ) the intertrial interval varied randomly ranging from $7.5-12.5 \mathrm{~s}$ to limit anticipation of the next trial. Peak-to-peak MEP amplitudes were measured in the single trials, and the mean at each time point was taken as a measure of corticospinal excitability (Ziemann and Hallett, 2007).

\section{MEASUREMENT OF SHORT-INTERVAL INTRACORTICAL INHIBITION (SICI) AND INTRACORTICAL FACILITATION (ICF)}

SICI and ICF were studied using an established paired-pulse TMS protocol (Kujirai et al., 1993; Ziemann et al., 1996). The two magnetic stimuli were given through the same figure-of-eight stimulating coil over the left $M 1_{\text {HAND }}$ and the effect of the subthreshold conditioning stimulus (CS) on the test MEP elicited by the subsequent suprathreshold test stimulus (TS) was investigated. SICI was assessed at an interstimulus interval (ISI) of $2.0 \mathrm{~ms}$ because at this interval SICI is not contaminated by shortinterval intracortical facilitation (SICF) (Peurala et al., 2008). At B0, the CS intensity was adjusted to produce approximately 50\% inhibition in order to provide highest sensitivity for detection of changes in SICI after $\mathrm{CB} \rightarrow \mathrm{M} 1$ PAS. The CS intensities ranged from $70 \%$ to $90 \%$ AMT in different individuals. This CS intensity was kept constant throughout the experiment. ICF was assessed in a subset of subjects in a separate block of trials at an ISI of $10 \mathrm{~ms}$. The CS intensities ranged from 75\% to 95\% AMT in different individuals to produce consistent test MEP facilitation as described in previous studies (Ziemann et al., 1996; Di Lazzaro et al., 2006). This CS intensity was kept constant throughout the experiment. The TS intensity was adjusted to elicit $\mathrm{MEP}_{1 \mathrm{mV}}$ when unconditioned by $\mathrm{CS}$. Whenever $\mathrm{CB} \rightarrow \mathrm{M} 1$ PAS resulted in a change in test MEP amplitude, TS intensity was adjusted to provide $\mathrm{MEP}_{1 \mathrm{mV}}$ throughout all time points of the experiment. This is important because variation of test MEP amplitude influences expression of SICI and ICF (Sanger et al., 2001; Müller-Dahlhaus et al., 2008). Twelve paired CS-TS trials and twelve TS alone trials were recorded in pseudorandomized order. Conditional averages were calculated, and SICI and ICF were expressed by the mean conditioned MEP amplitude as a percentage of the unconditioned mean (Kujirai et al., 1993).

\section{MEASUREMENT OF CEREBELLO-MOTOR CORTEX INHIBITION (CBI)}

CBI was studied using an established paired-coil protocol (Ugawa et al., 1995). Individual AMT of the descending corticospinal tract was determined by placing a double cone coil (inner diameter of each wing, $110 \mathrm{~mm}$; Magstim Co., UK) over the inion with the coil junction oriented vertically and to induce a downward electric current in the underlying tissue. For the CBI measurements, the center of the coil was moved rightwards off the midpoint by $3 \mathrm{~cm}$ along a line between the inion and the right mastoid process, and the coil orientation was rotated by $180^{\circ}$ to induce an upward electric current in the right cerebellar hemisphere (Figure 2) (Ugawa et al., 1995; Daskalakis et al., 2004). The conditioning stimulus (CS) was delivered through this coil, and the intensity was set to 95\% AMT (as determined over the inion) and kept constant throughout the experiment. This low intensity was chosen to minimize confounding effects due to direct brainstem stimulation (Ugawa et al., 1995; Fisher et al., 2009). The test stimulus (TS) was delivered through a figure-of-eight coil placed over the left $M 1_{\text {HAND }}$ area and, whenever necessary, its intensity was adjusted throughout the four time points of the experiment (B0, P0, P30, and P60) to elicit an unconditioned test
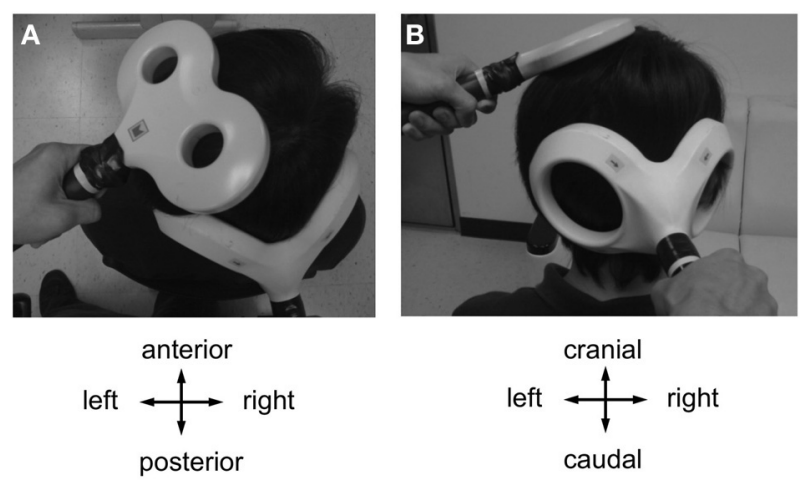

FIGURE 2 | Coil positions for cerebellar-motor cortical inhibition (CBI) and $C B \rightarrow$ M1 PAS (A, view from above; $B$, view from behind). A double cone coil was placed over the midpoint of the inion and the right mastoid process for stimulation of the right lateral cerebellum. The coil was oriented to induce an upward current in the cerebellum. A figure-of-eight coil was used to stimulate the left M1 HAND. The coil was oriented to induce a current in M1 directed from posterior-lateral to antero-medial. Motor-evoked potentials were recorded from the first dorsal interosseous muscle of the right hand. 
MEP in the right FDI of $0.6-0.8 \mathrm{mV}$ in peak-to-peak amplitude $\left(\mathrm{MEP}_{0.7 \mathrm{mV}}\right)$. Compared to the SICI and ICF measurements, a slightly lower test MEP amplitude was chosen because CBI was found to be more significant at this lower amplitude (Ugawa et al., 1995; Pinto and Chen, 2001; Daskalakis et al., 2004). CBI was assessed at an ISI of $7 \mathrm{~ms}$ which provided clear MEP inhibition in previous studies (Ugawa et al., 1995; Pinto and Chen, 2001), and is not affected by potential contamination from an MEP inhibition at longer ISIs induced by stimulation of peripheral nerve afferents (Werhahn et al., 1996). Twelve CS-TS trials and twelve TS alone trials alone were recorded in pseudo-randomized order. Conditional averages were calculated, and CBI was expressed by the mean conditioned MEP amplitude as a percentage of the unconditioned mean (Ugawa et al., 1995).

\section{CEREBELLUM TO MOTOR CORTEX PAIRED ASSOCIATIVE STIMULATION (CB $\rightarrow$ M1 PAS)}

Maximal CBI occurs when TS over $\mathrm{M}_{\text {HAND }}$ is given $6-7 \mathrm{~ms}$ after a CS over the contralateral cerebellum (Ugawa et al., 1995). Therefore, an ISI of 6-7 ms would result in arrival of the afferent signal elicited by the cerebellar CS at the same time in M1 HAND when TS of the M1 $1_{\text {HAND }}$ generates actions potentials in excitatory interneurons and corticospinal neurons, ISIs longer than $7 \mathrm{~ms}$ would result in arrival of the afferent signal before TS-induced actions potentials in $\mathrm{M} 1_{\mathrm{HAND}}$, and this order of events in the $M 1_{\text {HAND }}$ would be reversed if ISIs shorter than $6 \mathrm{~ms}$ were applied. According to other PAS protocols that have paired electrical stimulation of a peripheral nerve (Stefan et al., 2000, 2002; Wolters et al., 2003; Ziemann et al., 2004; Müller-Dahlhaus et al., 2010) or a magnetic CS to the supplementary motor area (Arai et al., 2011) with TMS of M1 HAND, and according to the principles of spike-timing dependent bidirectional plasticity (Markram et al., 1997; Bi and Poo, 2001) repeated pairing at long intervals ( $\geq 6 \mathrm{~ms}$ ) are expected to result in LTP-like changes, while shorter intervals should lead to LTD-like changes. However, CS results in an inhibitory input to the $\mathrm{M} 1_{\mathrm{HAND}}$, most likely through activation of Purkinje cells, the principal inhibitory neurons of the cerebellar hemispheres (Ugawa et al., 1995). STDP of inhibitory circuits has not been studied well in basic experiments (Lamsa et al., 2010). Therefore, it is difficult to predict the outcome of the present experiments. It might be speculated that LTP-like change of this inhibitory input leads to a long-term MEP decrease (due to strengthened inhibitory control of corticospinal cells), and vice versa, LTD-like change to a long-term MEP increase.

In separate sessions, $\mathrm{CB} \rightarrow \mathrm{M} 1$ PAS was applied at one of four different ISIs: $10 \mathrm{~ms}$ (13 subjects), $6 \mathrm{~ms}$ (6 subjects), $2 \mathrm{~ms}$ (13 subjects) or trial-by-trial randomly alternating ISIs of $10 \mathrm{~ms}$ and $2 \mathrm{~ms}$ (9 subjects) (Figure 1). The randomly alternating protocol was developed in our group originally for the conventional PAS protocol (i.e., pairing of electrical peripheral nerve stimulation with TMS of the contralateral M1 $1_{\text {HAND }}$ ) where it did not induce any significant change of MEP amplitude (Müller et al., 2007; Lu et al., 2009). The intensity of CS was kept at 95\% AMT determined over the inion. A total of $120 \mathrm{CS}$-TS pairs were delivered at a frequency of $0.25 \mathrm{~Hz}$ in each of the sessions (i.e., the duration of $\mathrm{CB} \rightarrow \mathrm{M} 1$ PAS was $8 \mathrm{~min}$ ). Several subjects participated in more than one $\mathrm{CB} \rightarrow \mathrm{M} 1$ PAS condition. In these cases, the order of $\mathrm{CB} \rightarrow \mathrm{M} 1$
PAS conditions was pseudo-randomized and the minimum interval between two successive sessions in a given subject was 5 days in order to avoid interactions between sessions.

\section{DATA ANALYSIS AND STATISTICS}

Statistics were performed with IBM SPSS (Version 20). Data are presented as means \pm SEM if not stated otherwise. For all tests a $P$ value of $<0.05$ was considered significant. Separate mixed repeated measures analyses of variance (rmANOVA) were used to test the effects of $\mathrm{CB} \rightarrow \mathrm{M} 1$ PAS on MEP amplitude, SICI, CBI, and ICF. The within subject effect was TIME (B0, $\mathrm{P} 0, \mathrm{P} 30$, and $\mathrm{P} 60)$, and the between-subject effect was PAS PROTOCOL $\left(\mathrm{CB} \rightarrow \mathrm{M} 1 \mathrm{PAS}_{10 \mathrm{~ms}}, \mathrm{CB} \rightarrow \mathrm{M} 1 \mathrm{PAS}_{6 \mathrm{~ms}}, \mathrm{CB} \rightarrow \mathrm{M} 1\right.$ $\mathrm{PAS}_{2 \mathrm{~ms}}$, and $\left.\mathrm{CB} \rightarrow \mathrm{M} 1 \mathrm{PAS}_{\text {Control }}\right)$. Conditional on a significant $F$ value, post-hoc comparisons were performed using pairedsample $t$-tests with Fisher's LSD correction for multiple comparisons. Violation of sphericity was checked with Mauchly's test and degrees of freedom were adjusted whenever Mauchly's $\mathrm{W}<0.05$ using the Greenhouse-Geisser correction.

\section{RESULTS}

None of the subjects experienced any noticeable adverse effects during or after the study. All subjects were cooperative throughout the experimental procedures. The grand averages ( \pm SD) across all sessions and subjects were for RMT: $43.4 \pm 5.8 \% \mathrm{MSO}$; AMT of $\mathrm{Ml}_{\mathrm{HAND}}$ : $33.4 \pm 4.2 \% \mathrm{MSO}$; $\mathrm{MEP}_{0.7 \mathrm{mV}}: 49.6 \pm 8.2 \%$ MSO; MEP $_{1 \mathrm{mV}}$ : $52.7 \pm 8.8 \%$ MSO; AMT over inion: $33.7 \pm$ $2.9 \%$ MSO. There were no differences between PAS protocols for any of these measures (all $P>0.5$ ).

\section{MEP AMPLITUDE}

There was a main effect of PAS PROTOCOL $\left[F_{(3,111)}=6.97\right.$, $P=0.001]$ and a significant PAS PROTOCOL $\times$ TIME interaction $\left[F_{(9,333)}=7.94, P<0.001\right]$ (Table 1, Figure 3A). Post-hoc comparisons showed significant differences of $\mathrm{CB} \rightarrow \mathrm{M} 1 \mathrm{PAS}_{10 \mathrm{~ms}}$

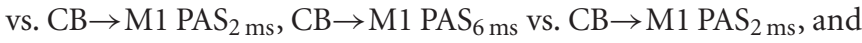

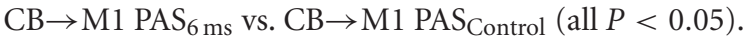

One-way rmANOVA of $\mathrm{CB} \rightarrow \mathrm{M} 1 \mathrm{PAS}_{10 \mathrm{~ms}}$ revealed a significant effect of TIME $\left[F_{(3,36)}=7.21, P=0.001\right]$, which was explained by significant MEP amplitude depression at $\mathrm{P} 0$ and P30 when compared to B0 (mean MEP amplitudes at B0: $1.09 \pm$ $0.04 \mathrm{mV}, \mathrm{P} 0: 0.86 \pm 0.06 \mathrm{mV}, \mathrm{P} 30: 0.83 \pm 0.07 \mathrm{mV}$, both $P<$ $0.01)$ but no longer at $60 \mathrm{~min}(\mathrm{P} 60)(1.00 \pm 0.06 \mathrm{mV}, P=0.11$; diamonds in Figure 3A). $\mathrm{CB} \rightarrow \mathrm{M1} \mathrm{PAS}{ }_{6 \mathrm{~ms}}$ also showed a significant effect of TIME $\left[F_{(3,15)}=6.46, P=0.005\right]$, which was explained by significant MEP amplitude depression at P0, P30, and P60 when compared to B0 (mean MEP amplitudes at B0:

Table 1 | Mixed rmANOVA of the $\mathrm{CB} \rightarrow \mathrm{M} 1$ PAS effects on MEP, SICl, and $\mathrm{CBI}$.

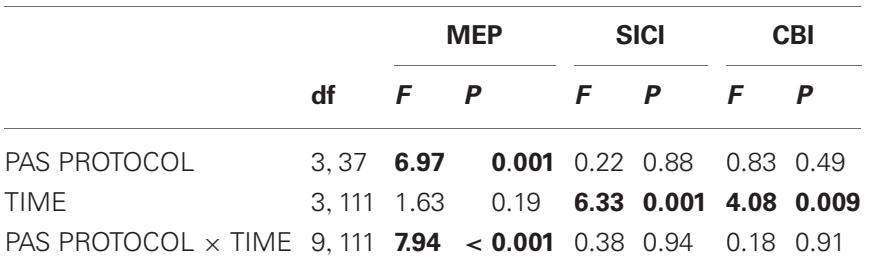



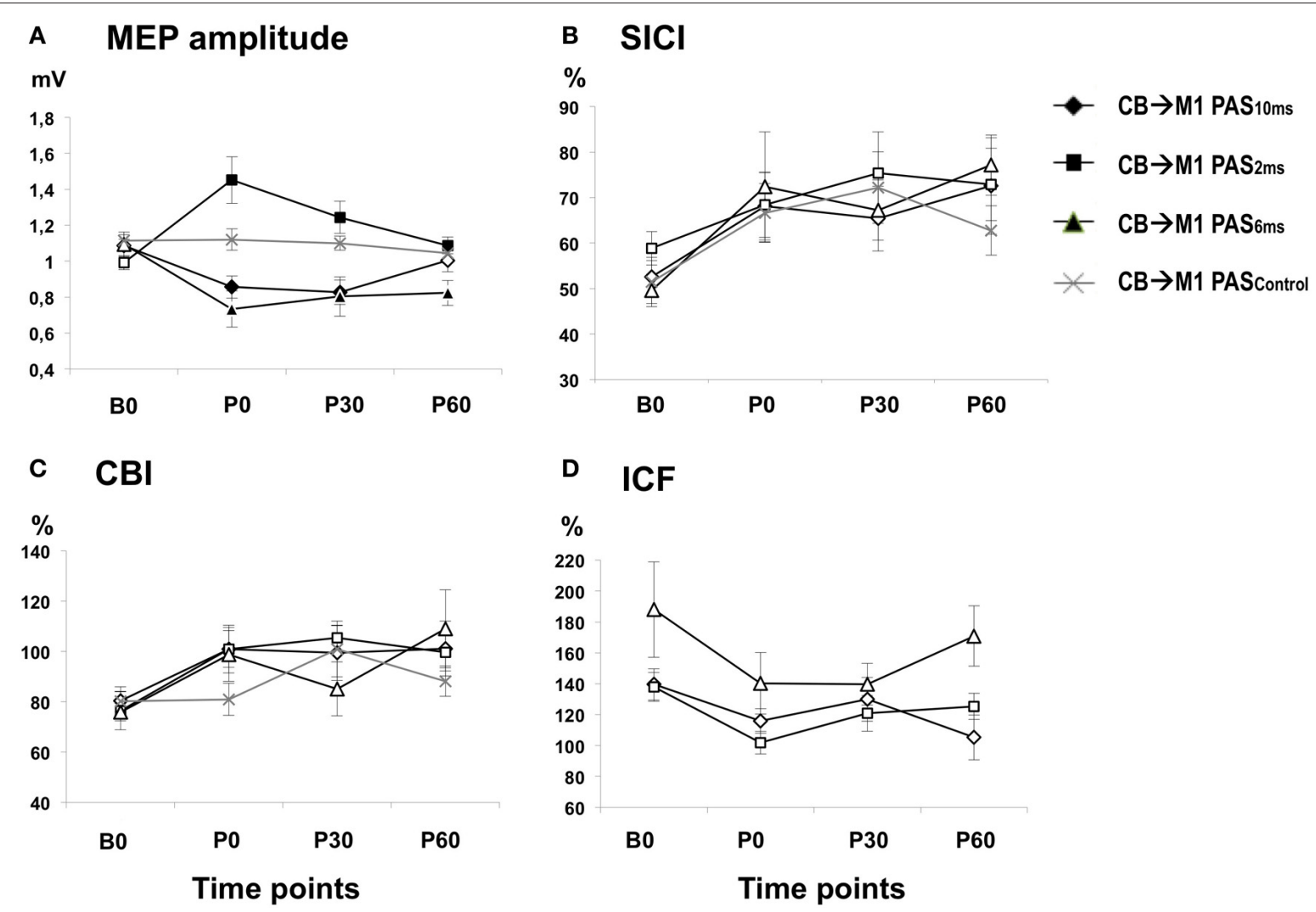

FIGURE 3 | (A) Means ( \pm SEM) of MEP amplitude (in $\mathrm{mV}$ ) at baseline (B0), immediately (P0), $30 \mathrm{~min}$ (P30) and $60 \mathrm{~min}$ (P60) after $\mathrm{CB} \rightarrow \mathrm{M} 1$ PAS (rhomboids: $\mathrm{CB} \rightarrow \mathrm{M} 1 \mathrm{PAS}_{10 \mathrm{~ms}}$; triangles: $\mathrm{CB} \rightarrow \mathrm{M} 1 \mathrm{PAS}_{6 \mathrm{~ms}}$; squares: $\mathrm{CB} \rightarrow \mathrm{M} 1 \mathrm{PAS}_{2 \mathrm{~ms}}$; crosses: $\mathrm{CB} \rightarrow \mathrm{M} 1 \mathrm{PAS}_{\text {Control }}$ ). Filled symbols denote significant differences in MEP amplitude after $\mathrm{CB} \rightarrow \mathrm{M} 1$ PAS compared to B0. Note significant MEP suppression at $P 0$ and $P 30$ after $C B \rightarrow M 1$ $\mathrm{PAS}_{10 \mathrm{~ms}}$ and at P0-P60 after $\mathrm{CB} \rightarrow \mathrm{M} 1 \mathrm{PAS}_{6 \mathrm{~ms}}$ but MEP potentiation at $\mathrm{P0}-\mathrm{P} 60$ after $\mathrm{CB} \rightarrow \mathrm{M} 1 \mathrm{PAS}_{2} \mathrm{~ms}$. In contrast, MEP amplitude remained unchanged after $\mathrm{CB} \rightarrow \mathrm{M} 1 \mathrm{PAS}_{\text {Control }}$ (B,C) Mean $\mathrm{SICl}$ and $\mathrm{CBI}$ (given as percentage of the conditioned MEP/unconditioned MEP). Otherwise, same conventions as in (A). $\mathrm{SICl}$ and $\mathrm{CBI}$ decreased significantly but non-specifically across all three PAS conditions, but this effect was no longer significant at the level of single time points and PAS conditions in the post-hoc tests. (D) Mean ICF (given as percentage of the conditioned MEP/unconditioned MEP). Same conventions as in (A). CB $\rightarrow$ M1 PAS had no significant effect on ICF.
$1.09 \pm 0.06 \mathrm{mV}, \mathrm{P} 0: 0.73 \pm 0.10 \mathrm{mV}, \mathrm{P} 30: 0.80 \pm 0.11 \mathrm{mV}, \mathrm{P} 60:$ $0.82 \pm 0.07 \mathrm{mV}$, all $P<0.05$; triangles in Figure $3 \mathrm{~A}) . \mathrm{CB} \rightarrow \mathrm{M} 1$ $\mathrm{PAS}_{2 \mathrm{~ms}}$ revealed a significant effect of $\operatorname{TIME}\left[F_{(3,36)}=8.61\right.$, $P<0.001$ ], which was explained by significant MEP amplitude potentiation at $\mathrm{P} 0, \mathrm{P} 30$, and $\mathrm{P} 60$ when compared to B0 (mean MEP amplitudes at B0: $0.99 \pm 0.04 \mathrm{mV}, \mathrm{P} 0: 1.45 \pm 0.13 \mathrm{mV}, \mathrm{P} 30$ : $1.24 \pm 0.09 \mathrm{mV}, \mathrm{P} 60: 1.09 \pm 0.05 \mathrm{mV}$, all $P<0.05$; squares in Figure 3A). Finally, $\mathrm{CB} \rightarrow \mathrm{M} 1 \mathrm{PAS}_{\text {Control }}$ showed no effect of TIME $\left[F_{(3,24)}=0.75, P=0.54\right.$; crosses in Figure 3A $]$.

There were no differences of mean MEP amplitudes at $\mathrm{B} 0$ across $\mathrm{CB} \rightarrow \mathrm{M} 1$ PAS protocols $\left(\mathrm{CB} \rightarrow \mathrm{M} 1 \mathrm{PAS}_{10 \mathrm{~ms}}: 1.09 \pm\right.$ $0.04 \mathrm{mV} ; \mathrm{CB} \rightarrow \mathrm{M} 1 \mathrm{PAS}_{6 \mathrm{~ms}}: 1.09 \pm 0.07 \mathrm{mV} ; \mathrm{CB} \rightarrow \mathrm{M} 1 \mathrm{PAS}_{6 \mathrm{~ms}}$ : $0.99 \pm 0.04 \mathrm{mV} ; \mathrm{CB} \rightarrow \mathrm{M} 1 \mathrm{PAS}_{\text {Control }}: 1.11 \pm 0.04 \mathrm{mV}$; all $P>$ $0.05)$ that could have accounted for the differential effects of the $\mathrm{CB} \rightarrow \mathrm{M} 1 \mathrm{PAS}$ protocols on MEP amplitude.

\section{SICI}

There was a main effect of $\operatorname{TIME}\left[F_{(3,11)}=6.33, P=0.001\right]$ but no significant effects of PAS PROTOCOL or of the TIME $\times$ PAS PROTOCOL interaction (both $P>0.8$ ) (Table 1). The effect of TIME was explained by a non-specific decrease of SICI at all post $\mathrm{CB} \rightarrow \mathrm{M} 1$ PAS time points compared to baseline (B0: $53.9 \pm 2.2 \%$, P0: $68.5 \pm 3.9 \%$, P $30: 70.3 \pm 4.2 \%$, P $60: 71.2 \pm$ 4.5\%; all $P<0.005$; Figure 3B). However, this effect of TIME was weak and no longer significant when tested separately for the different PAS protocols (all $P>0.05$ ). SICI at time point B0 did not differ between PAS protocols $\left(\mathrm{CB} \rightarrow \mathrm{M} 1 \mathrm{PAS}_{10 \mathrm{~ms}}\right.$ : $52.6 \pm 4.3 \% ; \mathrm{CB} \rightarrow \mathrm{M}_{1} \mathrm{PAS}_{6 \mathrm{~ms}}: 49.6 \pm 3.6 \% ; \mathrm{CB} \rightarrow \mathrm{M} 1 \mathrm{PAS}_{2 \mathrm{~ms}}$ : $58.8 \pm 3.7 \%$; $\mathrm{CB} \rightarrow \mathrm{M} 1 \mathrm{PAS}_{\text {Control }}: 51.4 \pm 5.7 \%$; all $P>0.15$, Figure 3B). There were no effects of TIME, PAS PROTOCOL or of the TIME $\times$ PAS PROTOCOL interaction on unconditioned test MEP amplitude (all $P>0.2$ ), indicating that maintenance of $\mathrm{MEP}_{1 \mathrm{mV}}$ across time points and PAS protocols (grand average, $0.95 \pm 0.23 \mathrm{mV}$ ) was successfully achieved during the SICI measurements.

\section{CBI}

There was a main effect of TIME $\left[F_{(3,111)}=4.08, P=0.009\right]$ but no effects of PAS PROTOCOL or of the TIME $\times$ PAS PROTOCOL interaction on CBI (both $P>0.4$ ) (Table 1). The effect of TIME was explained by a non-specific decrease of CBI at all post $\mathrm{CB} \rightarrow \mathrm{M} 1$ PAS time points compared to baseline (B0: 
$78.4 \pm 3.1 \%$, P0: $96.3 \pm 4.5 \%$, P30: $99.6 \pm 5.0 \%$, P60: $99.0 \pm$ 4.9\%; all $P<0.01$; Figure 3C). However, this effect of TIME was weak and no longer significant when tested separately for the different PAS protocols (all $P>0.05$ ). CBI at time point B0 did not differ between PAS protocols $\left(\mathrm{CB} \rightarrow \mathrm{M} 1 \mathrm{PAS}_{10 \mathrm{~ms}}\right.$ :

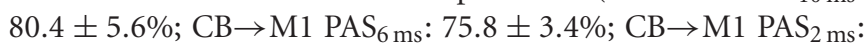
$76.5 \pm 7.6 \%$; $\mathrm{CB} \rightarrow \mathrm{M} 1 \mathrm{PAS}_{\text {Control }}: 80.1 \pm 5.0 \%$; all $P>0.5$, Figure 3C). There were no effects of TIME, PAS PROTOCOL or the TIME $\times$ PAS PROTOCOL interaction on unconditioned test MEP amplitude (all $P>0.05$ ), indicating that maintenance of $\mathrm{MEP}_{0.7 \mathrm{mV}}$ across time points and PAS protocols (grand average: $0.81 \pm 0.24 \mathrm{mV}$ ) was successfully achieved during the CBI measurements.

\section{ICF}

There were no effects of TIME $\left[F_{(3,33)}=2.36, P=0.08\right]$, PAS PROTOCOL $\left[F_{(2,11)}=4.01, P=0.06\right]$ or of the TIME $\times$ PAS PROTOCOL interaction $\left[F_{(6,33)}=0.63, P=0.70\right]$ on ICF (Figure 3D). ICF at time point B0 did not differ between PAS Protocols (all $P>0.2$, Figure 3D). There were no effects of TIME, PAS PROTOCOL or the TIME $\times$ PAS PROTOCOL interaction on unconditioned test MEP amplitude (all $P>0.05$ ), indicating that maintenance of $\mathrm{MEP}_{1 \mathrm{mV}}$ across time points and PAS protocols (grand average: $0.96 \pm 0.26 \mathrm{mV}$ ) was successfully achieved during the ICF measurements.

\section{DISCUSSION}

This study demonstrates, to the best of our knowledge for the first time, bidirectional long-term (> $30 \mathrm{~min}$ ) STDP-like plasticity of MEP amplitude induced by associative stimulation of the cerebello-dentato-thalamo-M1 pathway and the corticospinal output network in $\mathrm{M} 1: \mathrm{CB} \rightarrow \mathrm{M} 1 \mathrm{PAS}_{2 \mathrm{~ms}}$ resulted in an increase in corticospinal excitability indexed by MEP amplitude, while $\mathrm{CB} \rightarrow \mathrm{M} 1 \mathrm{PAS}_{6 \mathrm{~ms}}$ and $\mathrm{CB} \rightarrow \mathrm{M} 1 \mathrm{PAS}_{10 \mathrm{~ms}}$ resulted in a MEP decrease, and $\mathrm{CB} \rightarrow \mathrm{M} 1 \mathrm{PAS}_{\text {Control }}$ in no change. The data extend previous studies showing bidirectional STDPlike plasticity in M1 when focal TMS of the M1 HAND was paired with conditioning stimulation of a peripheral nerve (Stefan et al., 2000; Wolters et al., 2003) or with another TMS pulse applied to the ipsilateral ventral premotor cortex (Buch et al., 2011), suggesting that STDP-like plasticity in human M1 is a generally operating principle for various afferent inputs.

The "classical" PAS protocol to induce STDP-like plasticity employs repeated pairing of electrical stimulation of the median nerve with focal TMS of the contralateral M1 HAND (Stefan et al., 2000; Wolters et al., 2003; Ziemann et al., 2004; Müller et al., 2007) (for review, Müller-Dahlhaus et al., 2010). The ISI between the peripheral electrical stimulus and TMS of M1 HAND is critical for the expression of LTP-like vs. LTD-like changes in MEP amplitude: if the ISI is equal or longer than the individual latency of the earliest cortical component (N20) of the median nerve somatosensory-evoked potential, then a long-lasting (typically $>30 \mathrm{~min}$ ) LTP-like increase in MEP amplitude occurs in the majority of subjects. In contrast, ISIs that is shorter by 5-15 ms than the N20 latency result in a LTD-like MEP decrease
(Müller-Dahlhaus et al., 2010). If the afferent volley from peripheral nerve stimulation produced excitatory postsynaptic potentials (EPSPs) in the motor cortical output neurons then these EPSPs would precede action potential generation in corticospinal cells by TMS with the longer ISIs, while the order of these events would be reversed with the shorter ISIs. The resulting bidirectional M1 LTP/D-like plasticity is strongly reminiscent to STDP studied in neuronal slices or cultures where the temporal order of repeated pairing of EPSPs and action potentials determines the direction of synaptic plasticity (Markram et al., 1997; Bi and Poo, 2001; Dan and Poo, 2004). However, one critical issue with the classical PAS protocol is that the afferent electrical stimulation to the peripheral nerve causes MEP inhibition at intervals that are typically associated with LTP-like plasticity. This MEP inhibition has been termed short-latency afferent inhibition (SAI) and is mediated by cortical inhibitory interneurons (Tokimura et al., 2000; Di Lazzaro et al., 2007). It was also demonstrated that the presence of SAI causes a short-lasting decrease of SICI (Stefan et al., 2002; Alle et al., 2009). Since the level of local inhibition exerts a powerful role in regulating synaptic plasticity in M1 (Castro-Alamancos et al., 1995; Hess et al., 1996; Ziemann et al., 1998; Heidegger et al., 2010), this disinhibition (i.e., the decrease of SICI) may be crucial for an LTP-like effect to occur in the presence of SAI (Stefan et al., 2002). Another recently established PAS protocol is applied to the interhemispheric connection between the two M1 (Koganemaru et al., 2009; Rizzo et al., 2009). An ISI of $8 \mathrm{~ms}$ between TMS of the conditioning left M1 $1_{\text {HAND }}$ and TMS of the conditioned right $\mathrm{M} 1_{\text {HAND }}$ results in a LTPlike MEP increase in the right $\mathrm{M} 1_{\mathrm{HAND}}$ and a concomitant (but not correlated) decrease of short-latency interhemispheric inhibition (SIHI) (Rizzo et al., 2009). Similar to the classical PAS protocol, the paradox is that the LTP-like effect occurs although the conditioning M1 stimulation causes MEP inhibition in the conditioned M1 HAND in the form of SIHI (Ferbert et al., 1992; Di Lazzaro et al., 1999). However, triple-pulse TMS experiments revealed that the presence of SIHI reduces SICI (Daskalakis et al., 2002; Müller-Dahlhaus et al., 2008). Again, this disinhibition might be critical to permit the LTP-like effect to occur in this protocol.

Similar to those studies, $\mathrm{CB}$ conditioning stimulation also decreases MEP amplitude in the contralateral M1 HAND, termed cerebellar-motor inhibition (CBI) (Ugawa et al., 1995; Werhahn et al., 1996), and the presence of CBI reduces SICI (Daskalakis et al., 2004). However, in contrast to the classical and interhemispheric PAS protocols, $\mathrm{CB} \rightarrow \mathrm{M} 1 \mathrm{PAS}_{10 \mathrm{~ms}}$ and $\mathrm{CB} \rightarrow \mathrm{M} 1$ $\mathrm{PAS}_{6 \mathrm{~ms}}$ resulted in LTD-like MEP amplitude decrease although the $\mathrm{CB}$ conditioning effect reached $\mathrm{M} 1$ at the same time or prior to $\mathrm{M} 1_{\mathrm{HAND}}$ stimulation, given a $\mathrm{CB} \rightarrow \mathrm{M} 1$ conduction time of 5-6 ms (Ugawa et al., 1995; Werhahn et al., 1996; Pinto and Chen, 2001).

We can currently only speculate about the reasons for this apparent discrepancy. One important difference of $\mathrm{CB} \rightarrow \mathrm{M} 1$ PAS compared to the classical and interhemispheric PAS protocols is that TMS over the lateral cerebellum likely activates Purkinje cells, i.e., the major inhibitory interneurons of the cerebellum (Ugawa et al., 1995). In contrast, the conditioning pulses in the other PAS protocols activate primarily excitatory pathways, 
namely mixed or cutaneous nerve fibers in the classical PAS protocol (Tokimura et al., 2000) and glutamatergic interhemispheric fibers in the interhemispheric PAS protocol (Ferbert et al., 1992) that project onto inhibitory interneurons within M1. The Purkinje cells inhibit deep cerebellar nuclei, which facilitate tonically the contralateral M1 through the dentatothalamo-M1 pathway (Allen and Tsukahara, 1974; Thach, 1987; Middleton and Strick, 1998). Therefore, activation of the Purkinje cells leads to M1 disfacilitation. Repeated TMS of M1 at a time when $\mathrm{CB}$ conditioning stimulation has inhibited this tonically active pathway should lead to Hebbian LTD-like MEP decrease, similar to LTD induced in hippocampal slices when a highfrequency conditioning input was negatively correlated in time with a test input (Stanton and Sejnowski, 1989). The LTP-like MEP increase after $\mathrm{CB} \rightarrow \mathrm{M} 1 \mathrm{PAS}_{2 \mathrm{~ms}}$ implies a reversal of the order of these events in M1, i.e., action potential generation in $\mathrm{M} 1$ corticospinal cells regularly preceded the disfacilitation of the dentato-thalamo-M1 projection. This may have caused a transient strengthening of this tonic input because it was active above average at the time of TMS-induced action potential generation.

Our data are compatible with two $1 \mathrm{~Hz}$ repetitive TMS (rTMS) studies of the lateral cerebellum, which demonstrated an increase in MEP amplitude (Oliveri et al., 2005; Fierro et al., 2007). Low-frequency rTMS leads to depression of excitability of the stimulated brain area (Chen et al., 1997; Ziemann et al., 2008). Therefore, the putative depression of Purkinje cell excitability would lead to reduced inhibitory regulation of the dentatethalamo-M1 pathway and consequently to increased tonic excitatory input to M1.

At a first glance, it might be surprising that $\mathrm{CBI}$ did not change accordingly, i.e., decreased after $\mathrm{CB} \rightarrow \mathrm{M} 1 \mathrm{PAS}_{10 \mathrm{~ms}}$ and $\mathrm{CB} \rightarrow \mathrm{M} 1$ $\mathrm{PAS}_{6 \mathrm{~ms}}$ and increased after $\mathrm{CB} \rightarrow \mathrm{M1} \mathrm{PAS}_{2 \mathrm{~ms}}$. This lack of change in the strength of the stimulated conditioning pathway is very similar to classical PAS where SAI representing motor cortical inhibition mediated by an ascending central cholinergic projection (Di Lazzaro et al., 2000) does not change (Stefan et al., 2002; Hamada et al., 2012).

Other recent studies demonstrated a significant bidirectional change of CBI after anodal (CBI increase) vs. cathodal transcranial direct current stimulation (CBI decrease) of the lateral cerebellum (Galea et al., 2009), or a reduction of CBI after $1 \mathrm{~Hz}$ rTMS or continuous theta-burst stimulation (Popa et al., 2009) without changes in MEP amplitude. Together, these findings indicate that the modifications of M1 excitability (indexed by MEP amplitude) and CBI are often dissociated.

The non-specific decrease in SICI observed after all four PAS protocols (cf. Figure 3B) was unrelated to the bidirectional modification of MEP amplitude. SICI remained unaffected by $1 \mathrm{~Hz}$ rTMS of the lateral cerebellum (Oliveri et al., 2005; Fierro et al., 2007). Thus, it is unlikely that the low-frequency $(0.25 \mathrm{~Hz})$ stimulation of the cerebellum per se employed in all three PAS protocols of our study was responsible for the SICI change. One possible explanation is provided by the observation that STDP protocols in rat somatosensory cortex resulted always in LTD at excitatory synapses from pyramidal cells onto fast-spiking inhibitory interneurons, irrespective of the interval between the pre- and postsynaptic spikes (Lu et al., 2007). Fastspiking interneurons are the parvalbumin positive basket cells and chandelier cells (Kawaguchi and Kondo, 2002), which are currently thought to mediate SICI (Ilic et al., 2002; Di Lazzaro et al., 2007).

$\mathrm{CB} \rightarrow \mathrm{M} 1$ PAS did not affect ICF in a STDP-like manner (Figure 3D). Previous studies on modulation of ICF by CB stimulation showed inconsistent results. One Hz rTMS resulted either in an ICF increase (Oliveri et al., 2005) or an ICF decrease (Fierro et al., 2007), with a concomitant increase in MEP amplitude (Fierro et al., 2007) or the problem that the observed changes in ICF were not controlled for increases in test MEP amplitude (Oliveri et al., 2005). Continuous theta-burst stimulation of the lateral cerebellum resulted in MEP amplitude decrease but no change in ICF, while intermittent theta-burst stimulation led to an increase in MEP amplitude but a decrease in ICF (Koch et al., 2008). In summary, this corroborates the notion of dissociable physiological mechanisms that underlie MEP amplitude vs. ICF (Di Lazzaro et al., 2006, 2011; Ni et al., 2011). As with the classical PAS protocol (Stefan et al., 2000; MüllerDahlhaus et al., 2010) the present findings suggest that MEP amplitude is the most suitable marker to demonstrate STDPlike plasticity. This is most likely explained by the fact that MEP amplitude directly tests synaptic excitability of exactly those excitatory connections onto the corticospinal pathway (Hallett, 2007; Di Lazzaro et al., 2008), which are stimulated during PAS and undergo change in synaptic strength in accord with the principles of STDP.

It is a long-held concern that the magnetic stimulation of the lateral cerebellum with a large double cone coil (Figure 2) is nonspecific and may, in addition, excite sensory afferent fibers in the brachial plexus or the spinal dorsal nerve roots (Werhahn et al., 1996). This is, however, very unlikely to explain the present results because excitation of fast-conducting somatosensory afferents should behave like classical PAS (Stefan et al., 2000; Wolters et al., 2003), i.e., the $\mathrm{CB} \rightarrow \mathrm{M} 1 \mathrm{PAS}_{10 \mathrm{~ms}}$ and $\mathrm{CB} \rightarrow \mathrm{M} 1$ $\mathrm{PAS}_{6 \mathrm{~ms}}$ protocols should have resulted in an LTP-like increase of MEP amplitude and the $\mathrm{CB} \rightarrow \mathrm{M} 1 \mathrm{PAS}_{2 \mathrm{~ms}}$ in a LTD-like MEP decrease, while we observed MEP modifications in the opposite directions.

Another concern is direct excitation of the pyramidal tract at the level of the foramen magnum (Ugawa et al., 1994; Fisher et al., 2009) and antidromic propagation of the elicited action potentials via recurrent collaterals into the M1 circuitry (Stefanis and Jasper, 1964). Again, this is a highly unlikely scenario for the following reasons. The threshold was determined with conditions which typically result in lowest threshold values, i.e., with the coil centered over the midline at the inion level and with a downward directed induced current (Ugawa et al., 1994). For stimulation of the lateral cerebellum, a subthreshold stimulus intensity of 95\% AMT was used, the coil was lateralized by $3 \mathrm{~cm}$ and an upward directed induced current was applied. These are the optimal conditions for eliciting CBI in the absence of direct stimulation of the corticospinal tract (Ugawa et al., 1995; Pinto and Chen, 2001; Fisher et al., 2009). 
In summary, our findings suggest the possibility to induce STDP-like plasticity in human M1 by PAS of the contralateral cerebellum and M1. This bidirectional modification of M1 excitability may prove useful for correcting abnormal M1 excitability caused by cerebellar disease. Future studies may investigate the behavioral significance of this plasticity, in particular with respect to motor performance and motor adaptation.

\section{REFERENCES}

Alle, H., Heidegger, T., Krivanekova, L., and Ziemann, U. (2009). Interactions between short-interval intracortical inhibition and shortlatency afferent inhibition in human motor cortex. J. Physiol. 587, 5163-5176.

Allen, G. I., and Tsukahara, N. (1974). Cerebrocerebellar communication systems. Physiol. Rev. 54, 957-1006.

Arai, N., Müller-Dahlhaus, F., Murakami, T., Bliem, B., Lu, M. K., Ugawa, Y., and Ziemann, U. (2011). State-dependent and timing-dependent bidirectional associative plasticity in the human SMA-M1 network. J. Neurosci. 31, 15376-15383.

Bi, G., and Poo, M. (2001). Synaptic modification by correlated activity: Hebb's postulate revisited. Аnnu. Rev. Neurosci. 24, 139-166.

Brooks, V. B., and Thach, W. T. (1981). "Cerebellar control of posture and movement," in handbook of Physiology, Section I: The Nervous System. Vol. H: Motor Control ed V. B. Brooks (Bethesda: Oxford University Press), 877-946.

Buch, E. R., Johnen, V. M., Nelissen, N., O'shea, J., and Rushworth, M. F. (2011). Noninvasive associative plasticity induction in a corticocortical pathway of the human brain. $J$. Neurosci. 31, 17669-17679.

Castro-Alamancos, M. A., Donoghue, J. P., and Connors, B. W. (1995). Different forms of synaptic plasticity in somatosensory and motor areas of the neocortex. J. Neurosci. 15, 5324-5333.

Chen, R., Classen, J., Gerloff, C., Celnik, P., Wassermann, E. M., Hallett, M., and Cohen, L. G. (1997). Depression of motor cortex excitability by low-frequency transcranial magnetic stimulation. Neurology 48, 1398-1403.

Dan, Y., and Poo, M. M. (2004). Spike timing-dependent plasticity of neural circuits. Neuron 44, 23-30.

Daskalakis, Z. J., Christensen, B. K., Fitzgerald, P. B., Roshan, L., and Chen, R. (2002). The mechanisms of interhemispheric inhibition in the human motor cortex. J. Physiol. 543, 317-326.
Daskalakis, Z. J., Paradiso, G. O., Christensen, B. K., Fitzgerald, P. B., Gunraj, C., and Chen, R. (2004). Exploring the connectivity between the cerebellum and motor cortex in humans. J. Physiol. 557, 689-700.

Del Olmo, M. F., Cheeran, B., Koch, G., and Rothwell, J. C. (2007). Role of the cerebellum in externally paced rhythmic finger movements. J. Neurophysiol. 98, 145-152.

Di Lazzaro, V., Oliviero, A., Profice, P., Insola, A., Mazzone, P., Tonali, P., and Rothwell, J. C. (1999). Direct demonstration of interhemispheric tex produced by transcranial magnetic stimulation. Exp. Brain Res. $124,520-524$.

Di Lazzaro, V., Oliviero, A., Profice, P., Pennisi, M. A., Di Giovanni, S., Zito, G., Tonali, P., and Rothwell, J. C. (2000). Muscarinic receptor blockade has differential effects on the excitability of intracortical circuits in human motor cortex. Exp. Brain Res. 135, 455-461.

Di Lazzaro, V., Pilato, F., Dileone, M., Profice, P., Ranieri, F., Ricci, V., Bria, P., Tonali, P. A., and Ziemann, U. (2007). Segregating two inhibitory circuits in human motor cortex at the level of GABAA receptor subtypes: a TMS study. Clin. Neurophysiol. 118, 2207-2214.

Di Lazzaro, V., Pilato, F., Oliviero, A., Dileone, M., Saturno, E., Mazzone, P., Insola, A., Profice, P., Ranieri, F., Capone, F., Tonali, P. A., and Rothwell, J. C. (2006). Origin of facilitation of motor-evoked potentials after paired magnetic stimulation: direct recording of epidural activity in conscious humans. J. Neurophysiol. 96, 1765-1771.

Di Lazzaro, V., Profice, P., Ranieri, F., Capone, F., Dileone, M., Oliviero, A., and Pilato, F. (2011). I-wave origin and modulation. Brain Stimul. doi: $\quad$ 10.1016/j.brs.2011.07.008. [Epub ahead of print].

Di Lazzaro, V., Ziemann, U., and Lemon, R. N. (2008). State of the art: physiology of transcranial motor cortex stimulation. Brain Stimul. 1, 345-362.

Ferbert, A., Priori, A., Rothwell, J. C., Day, B. L., Colebatch, J. inhibition of the human motor cor-

\section{ACKNOWLEDGMENTS}

The authors wish to thank Drs. Takenobu Murakami and Tonio Heidegger for valuable discussion. This work was supported by grants (to Ming-Kuei Lu) from the Taiwan Department of Health Clinical Trial and Research Center of Excellence (DOH101-TDB-111-004) and "Aim for the Top University Plan" of the National Chiao Tung University and Ministry of Education, Taiwan.

G., and Marsden, C. D. (1992). Interhemispheric inhibition of the human motor cortex. J. Physiol. (Lond.) 453, 525-546.

Fierro, B., Giglia, G., Palermo, A. Pecoraro, C., Scalia, S., and Brighina, F. (2007). Modulatory effects of $1 \mathrm{~Hz}$ rTMS over the cerebellum on motor cortex excitability. Exp. Brain Res. 176, 440-447.

Fisher, K. M., Lai, H. M., Baker, M. R., and Baker, S. N. (2009). Corticospinal activation confounds cerebellar effects of posterior fossa stimuli. Clin. Neurophysiol. 120, 2109-2113.

Galea, J. M., Jayaram, G., Ajagbe, L., and Celnik, P. (2009). Modulation of cerebellar excitability by polarityspecific noninvasive direct current stimulation. J. Neurosci. 29, 9115-9122.

Galea, J. M., Vazquez, A., Pasricha, N., Orban De Xivry, J. J., and Celnik, P. (2011). Dissociating the roles of the cerebellum and motor cortex during adaptive learning: the motor cortex retains what the cerebellum learns. Cereb. Cortex 21, 1761-1767.

Groiss, S. J., and Ugawa, Y. (2012). Cerebellar stimulation in ataxia. Cerebellum 2, 440-442.

Groppa, S., Oliviero, A., Eisen, A. Quartarone, A., Cohen, L. G., Mall, V., Kaelin-Lang, A., Mima, T., Rossi, S., Thickbroom, G. W., Rossini, P. M., Ziemann, U., Valls-Sole, J., and Siebner, H. R. (2012). A practical guide to diagnostic transcranial magnetic stimulation: report of an IFCN committee. Clin. Neurophysiol. 123, 858-882.

Hallett, M. (2007). Transcranial magnetic stimulation: a primer. Neuron 55, 187-199.

Hamada, M., Strigaro, G., Murase, N., Sadnicka, A., Galea, J. M., Edwards, M. J., and Rothwell, J. C. (2012). Cerebellar modulation of human associative plasticity. J. Physiol. 590, 2365-2374.

Heidegger, T., Krakow, K., and Ziemann, U. (2010). Effects of antiepileptic drugs on associative LTP-like plasticity in human motor cortex. Eur. J. Neurosci. 32, 1215-1222.
Hess, G., Aizenman, C. D., and Donoghue, J. P. (1996). Conditions for the induction of long-term potentiation in layer II/III horizontal connections of the rat motor cortex. J. Neurophysiol. 75, 1765-1778.

Hoffland, B. S., Bologna, M., Kassavetis, P., Teo, J. T., Rothwell, J. C., Yeo, C. H., Van De Warrenburg, B. P., and Edwards, M. J. (2012). Cerebellar theta burst stimulation impairs eyeblink classical conditioning. J. Physiol. 590, 887-897.

Hoover, J. E., and Strick, P. L. (1999). The organization of cerebellar and basal ganglia outputs to primary motor cortex as revealed by retrograde transneuronal transport of herpes simplex virus type 1 . J. Neurosci. 19, 1446-1463.

Ilic, T. V., Meintzschel, F., Cleff, U., Ruge, D., Kessler, K. R., and Ziemann, U. (2002). Short-interval paired-pulse inhibition and facilitation of human motor cortex: the dimension of stimulus intensity. J. Physiol. 545.1, 153-167.

Kawaguchi, Y., and Kondo, S. (2002). Parvalbumin, somatostatin and cholecystokinin as chemical markers for specific GABAergic interneuron types in the rat frontal cortex. J. Neurocytol. 31, 277-287.

Koch, G., Mori, F., Marconi, B., Codeca, C., Pecchioli, C., Salerno, S., Torriero, S., Lo Gerfo, E., Mir, P., Oliveri, M., and Caltagirone, C. (2008). Changes in intracortical circuits of the human motor cortex following theta burst stimulation of the lateral cerebellum. Clin. Neurophysiol. 119, 2559-2569.

Koganemaru, S., Mima, T., Nakatsuka, M., Ueki, Y., Fukuyama, H., and Domen, K. (2009). Human motor associative plasticity induced by paired bihemispheric stimulation. J. Physiol. 587, 4629-4644.

Kujirai, T., Caramia, M. D., Rothwell, J. C., Day, B. L., Thompson, P. D., Ferbert, A., Wroe, S., Asselman, P., and Marsden, C. D. (1993). Corticocortical inhibition in human motor cortex. J. Physiol. (Lond.) 471, 501-519.

Lamsa, K. P., Kullmann, D. M., and Woodin, M. A. (2010). Spike-timing 
dependent plasticity in inhibitory circuits. Front. Syn. Neurosci. 2:8. doi: 10.3389/fnsyn.2010.00008

Lu, J. T., Li, C. Y., Zhao, J. P., Poo, M. M., and Zhang, X. H. (2007). Spike-timing-dependent plasticity of neocortical excitatory synapses on inhibitory interneurons depends on target cell type. J. Neurosci. 27, 9711-9720.

Lu, M. K., Bliem, B., Jung, P., Arai, N., Tsai, C. H., and Ziemann, U. (2009). Modulation of preparatory volitional motor cortical activity by paired associative transcranial magnetic stimulation. Hum. Brain Mapp. 30, 3645-3656.

Markram, H., Lübke, J., Frotscher, M., and Sakmann, B. (1997). Regulation of synaptic efficacy by coincidence of postsynaptic APs and EPSPs. Science 275, 213-215.

Miall, R. C., and Christensen, L. O. (2004). The effect of rTMS over the cerebellum in normal human volunteers on peg-board movement performance. Neurosci. Lett. 371, 185-189.

Middleton, F. A., and Strick, P. L. (1998). Cerebellar output: motor and cognitive channels. Trends Cogn. Sci. 2, 348-354.

Müller-Dahlhaus, F., Ziemann, U., and Classen, J. (2010). Plasticity resembling spike-timing dependent synaptic plasticity: the evidence in human cortex. Front. Syn. Neurosci. 2:1-11. doi: 10.3389/fnsyn.2010.00034

Müller-Dahlhaus, J. F., Liu, Y., and Ziemann, U. (2008). Inhibitory circuits and the nature of their interactions in the human motor cortex a pharmacological TMS study. J. Physiol. 586, 495-514.

Müller, J. F. M., Orekhov, Y., Liu, Y., and Ziemann, U. (2007). Homeostatic plasticity in human motor cortex demonstrated by two consecutive sessions of paired associative stimulation. Eur. J. Neurosci. 25, 3461-3468.

Ni, Z., Gunraj, C., Wagle-Shukla, A., Udupa, K., Mazzella, F., Lozano, A. M., and Chen, R. (2011). Direct demonstration of inhibitory interactions between long interval intracortical inhibition and short interval intracortical inhibition. J. Physiol. 589, 2955-2962.

Oldfield, R. C. (1971). The assessment and analysis of handedness: the Edinburgh inventory. Neuropsychologia 9, 97-113.
Oliveri, M., Koch, G., Torriero, S., and Caltagirone, C. (2005). Increased facilitation of the primary motor cortex following $1 \mathrm{~Hz}$ repetitive transcranial magnetic stimulation of the contralateral cerebellum in normal humans. Neurosci. Lett. 376, 188-193.

Panouilleres, M., Neggers, S. F., Gutteling, T. P., Salemme, R., Stigchel, S. V., Van Der Geest, J. N., Frens, M. A., and Pelisson, D. (2012). Transcranial magnetic stimulation and motor plasticity in human lateral cerebellum: dual effect on saccadic adaptation. Hum. Brain Mapp. 33, 1512-1525.

Peurala, S. H., Müller-Dahlhaus, J. F. M., Arai, N., and Ziemann, U. (2008). Interference of shortinterval intracortical inhibition (SICI) and short-interval intracortical facilitation (SICF). Clin. Neurophysiol. 119, 2291-2297.

Pinto, A. D., and Chen, R. (2001). Suppression of the motor cortex by magnetic stimulation of the cerebellum. Exp. Brain Res. 140, 505-510.

Popa, T., Russo, M., and Meunier, S. (2009). Long-lasting inhibition of cerebellar output. Brain Stimul. 3, 161-169.

Rizzo, V., Siebner, H. S., Morgante, F., Mastroeni, C., Girlanda, P., and Quartarone, A. (2009). Paired associative stimulation of left and right human motor cortex shapes interhemispheric motor inhibition based on a Hebbian mechanism. Cereb. Cortex 19, 907-915.

Rossini, P. M., Barker, A. T., Berardelli, A., Caramia, M. D., Caruso, G., Cracco, R. Q., Dimitrijevic, M. R., Hallett, M., Katayama, Y., Lücking, C. H., Maertens De Noordhout, A., Marsden, C. D., Murray, N. M. F., Rothwell, J. C., Swash, M., and Tomberg, C. (1994). Noninvasive electrical and magnetic stimulation of the brain, spinal cord and roots: basic principles and procedures for routine clinical application. Report of an IFCN committee. Electroencephalogr. Clin. Neurophysiol. 91, 79-92.

Sanger, T. D., Garg, R. R., and Chen, R. (2001). Interactions between two different inhibitory systems in the human motor cortex. J. Physiol. 530(Pt 2), 307-317.

Shmuelof, L., and Krakauer, J. W. (2011). Are we ready for a natural history of motor learning? Neuron 72, 469-476.

Stanton, P. K., and Sejnowski, T. J. (1989). Associative long-term depression in the hippocampus induced by hebbian covariance. Nature 339, 215-218.

Stefan, K., Kunesch, E., Benecke, R., Cohen, L. G., and Classen, J. (2002). Mechanisms of enhancement of human motor cortex excitability induced by interventional paired associative stimulation. J. Physiol. 543, 699-708.

Stefan, K., Kunesch, E., Cohen, L. G., Benecke, R., and Classen, J. (2000). Induction of plasticity in the human motor cortex by paired associative stimulation. Brain 123, 572-584.

Stefanis, C., and Jasper, H. (1964). Recurrent collateral inhibition in pyramidal tract neurons. $J$. Neurophysiol. 27, 855-877.

Thach, W. T. (1987). Cerebellar inputs to motor cortex. Ciba Found Symp. 132, 201-220.

Tokimura, H., Di Lazzaro, V., Tokimura, Y., Oliviero, A., Profice, P., Insola, A., Mazzone, P., Tonali, P., and Rothwell, J. C. (2000). Short latency inhibition of human hand motor cortex by somatosensory input from the hand. J. Physiol. 523, 503-513.

Torriero, S., Oliveri, M., Koch, G., Caltagirone, C., and Petrosini, L. (2004). Interference of left and right cerebellar rTMS with procedural learning. J. Cogn. Neurosci. 16, 1605-1611.

Ugawa, Y., Uesaka, Y., Terao, Y., Hanajima, R., and Kanazawa, I. (1994). Magnetic stimulation of corticospinal pathways at the foramen magnum level in humans. Ann. Neurol. 36, 618-624.

Ugawa, Y., Uesaka, Y., Terao, Y., Hanajima, R., and Kanazawa, I. (1995). Magnetic stimulation over the cerebellum in humans. Ann. Neurol. 37, 703-713.

Werhahn, K. J., Taylor, J., Ridding, M., Meyer, B. U., and Rothwell, J. C. (1996). Effect of transcranial magnetic stimulation over the cerebellum on the excitability of human motor cortex. Electroencephalogr. Clin. Neurophysiol. 101, 58-66.

Wolters, A., Sandbrink, F., Schlottmann, A., Kunesch, E., Stefan, K., Cohen, L. G., Benecke, R., and Classen, J. (2003). A temporally asymmetric Hebbian rule governing plasticity in the human motor cortex. J. Neurophysiol. 89, 2339-2345.

Ziemann, U., and Hallett, M. (2007). "Basic neurophysiological studies with transcranial magnetic stimulation," in Transcranial Magnetic Stimulation in Clinical Psychiatry, eds M. S. George and R. H. Belmaker (Washington, DC: American Psychiatric Publishing, Inc.), 59-84.

Ziemann, U., Hallett, M., and Cohen, L. G. (1998). Mechanisms of deafferentation-induced plasticity in human motor cortex. J. Neurosci. 18, 7000-7007.

Ziemann, U., Ilic, T. V., Pauli, C., Meintzschel, F., and Ruge, D. (2004). Learning modifies subsequent induction of LTP-like and LTD-like plasticity in human motor cortex. J. Neurosci. 24, 1666-1672.

Ziemann, U., Paulus, W., Nitsche, M. A., Pascual-Leone, A., Byblow, W. D., Berardelli, A., Siebner, H. R., Classen, J., Cohen, L. G., and Rothwell, J. C. (2008). Consensus: motor cortex plasticity protocols. Brain Stimul. 1, 164-182.

Ziemann, U., Rothwell, J. C., and Ridding, M. C. (1996). Interaction between intracortical inhibition and facilitation in human motor cortex. J. Physiol. 496, 873-881.

Conflict of Interest Statement: The authors declare that the research was conducted in the absence of any commercial or financial relationships that could be construed as a potential conflict of interest.

Received: 15 April 2012; accepted: 31 August 2012; published online: 19 September 2012.

Citation: $\mathrm{Lu} \mathrm{M}-\mathrm{K}$, Tsai $\mathrm{C}-\mathrm{H}$ and Ziemann U (2012) Cerebellum to motor cortex paired associative stimulation induces bidirectional STDP-like plasticity in human motor cortex. Front. Hum. Neurosci. 6:260. doi: 10.3389/fnhum. 2012.00260

Copyright (c) $2012 \mathrm{Lu}, \quad$ Tsai and Ziemann. This is an open-access article distributed under the terms of the Creative Commons Attribution License, which permits use, distribution and reproduction in other forums, provided the original authors and source are credited and subject to any copyright notices concerning any third-party graphics etc. 